\title{
Exploring the Role of Demand Shifting in Oligopolistic Electricity Markets
}

\author{
Dimitrios Papadaskalopoulos, Yujian Ye, and Goran Strbac \\ Department of Electrical and Electronic Engineering \\ Imperial College London \\ London, UK \\ d.papadaskalopoulos08@imperial.ac.uk,yujian.ye11@imperial.ac.uk,g.strbac@imperial.ac.uk
}

\begin{abstract}
Previous work has demonstrated that the price elasticity of the demand side reduces electricity producers' ability to exercise market power. However, price elasticity cannot capture alone consumers' flexibility, as the latter mainly involves shifting of loads' operation in time. This paper provides for the first time qualitative and quantitative analysis of the value of demand shifting in mitigating market power by the generation side. An equilibrium programming model of the oligopolistic market setting is developed, taking into account the inter-temporal characteristics of demand shifting. The decision making process of each strategic producer is modelled through a bi-level optimization problem, which is solved after transforming it to a Mathematical Program with Equilibrium Constraints (MPEC). The market equilibria resulting from the interaction of multiple independent producers are determined by employing an iterative diagonalization method. Case studies on a test market with day-ahead horizon and hourly resolution quantitatively demonstrate the benefits of demand shifting in limiting generation market power, by employing relevant indexes from the literature.
\end{abstract}

Index Terms--Bi-level optimization, demand shifting, electricity markets, equilibrium programming, market power, mathematical program with equilibrium constraints.

\section{NOMENCLATURE}

\section{A. Indices}

$t \quad$ Index of time periods running from 1 to $T$

$i \quad$ Index of producers running from 1 to $I$

\section{B. Parameters}

$l_{i}^{G} \quad$ Price intercept of marginal cost curve of producer $i$ (£/MW)

$q_{i}^{G} \quad$ Slope of marginal cost curve of producer $i\left(£ / \mathrm{MW}^{2}\right)$

$g_{i}^{\max }$ Maximum power output limit of producer $i$ (MW)

$l_{t}^{D} \quad$ Price intercept of marginal benefit curve of demand at time period $t(£ / \mathrm{MW})$

$q_{t}^{D} \quad$ Slope of marginal benefit curve of demand at time period $t\left(£ / \mathrm{MW}^{2}\right)$ $d_{t}^{\max }$ Maximum power input limit of baseline demand at time period $t$ (MW)

$\alpha \quad$ Parameter determining the limit of demand shifting

C. Variables

$k_{i, t} \quad$ Strategic offer variable of producer $i$ at time period $t$

$k_{i-, t} \quad$ Strategic offer variables of producers other than $i$ at time period $t$

$g_{i, t} \quad$ Power output of producer $i$ at time period $t$ (MW)

$d_{t} \quad$ Baseline demand at time period $t$ (MW)

$d_{t}^{s h} \quad$ Change of demand at time period $t$ due to load shifting (MW)

$d_{t}^{\prime} \quad$ Demand at time period $t$ after load shifting (MW)

$\lambda_{t} \quad$ Market clearing price at time period $t(£ / \mathrm{MWh})$

\section{INTRODUCTION}

After the deregulation of the energy sector, electricity markets are better described in terms of oligopoly rather than perfect competition. In this setting, market participants do not necessarily act as price takers. In particular, electricity producers controlling a large share of the market are able to manipulate the electricity prices and increase their profits beyond the competitive equilibrium levels, by virtue of strategic offers. This exercise of market power results in loss of social welfare and increase in consumers' payments [1].

Previous work investigating the role of the demand side in this context has demonstrated that the self-price elasticity of demand reduces electricity producers' ability to exercise market power [2]-[7], as demand is reduced at high market prices and thus limits the volume of electricity sold by strategic producers. A theoretical explanation of this effect is presented in [2]-[3]. Authors in [4]-[5] employ a Supply Function Equilibrium (SFE) model to determine the oligopoly equilibria with different levels of demand's price elasticity and define a number of market power indexes in order to quantitatively analyse the impact of elasticity. In [6], the same authors model the effect of two demand response programs, namely time-of-use (TOU) pricing and economic load 
response program (ELRP), on the price elasticity of demand and subsequently assess their impacts on the extent of exercised market power. Finally, an agent-based electricity market model is employed in [7] to assess the benefits of different elasticity levels in limiting market power.

However, a large number of researchers have stressed that demand flexibility regarding electricity use cannot be fully captured through the concept of self-price elasticity. Instead of simply avoiding using their loads at high price levels, consumers are more likely to shift the operation of their loads from periods of higher prices to periods of lower prices [2], [8]-[11]. In other words, load reduction during certain periods is accompanied by a load recovery effect during preceding or succeeding periods. Although numerous studies have investigated the impacts of this time-shifting flexibility of demand [8]-[11] on various aspects of power system operation and planning, its role in oligopolistic markets has not been investigated yet.

This paper aims at filling this knowledge gap by providing both qualitative and quantitative evidence of the value of demand shifting in mitigating market power by the generation side. Quantitative analysis is facilitated through a multi-period equilibrium programming model of the oligopolistic market setting. The decision making process of each strategic producer is modelled through a bi-level optimization problem. The upper level represents the profit maximization problem of the producer and the lower level represents endogenously the market clearing process, taking into account the inter-temporal characteristics of demand shifting. This bi-level problem is solved after transforming it to a Mathematical Program with Equilibrium Constraints (MPEC). The market equilibria resulting from the interaction of multiple independent producers are determined by employing an iterative diagonalization method. Case studies on a test market with day-ahead horizon and hourly resolution, quantitatively demonstrate the benefits of demand shifting in mitigating generators' market power, resorting to relevant market power indexes from the literature [4]-[5].

The rest of this paper is organised as follows. Section II outlines models of generation and demand market participants. Section III provides a theoretical explanation of the beneficial impact of demand shifting on the extent of market power exercised by the generation side. Section IV details the formulation of the equilibrium programming model for the oligopolistic market. Case studies and illustrative results are presented in Section V. Finally, Section VI concludes.

\section{MODELLING MARKET PARTICIPANTS}

\section{A. Strategic Electricity Producers}

For brevity reasons and without loss of generality, it is assumed that each electricity producer $i$ owns a single generation unit, the quadratic cost function, linear marginal cost function and output limits of which are expressed by (1), (2) and (3) respectively:

$C_{i, t}\left(g_{i, t}\right)=l_{i}^{G} g_{i, t}+q_{i}^{G}\left(g_{i, t}\right)^{2}$

$C_{i, t}^{\prime}\left(g_{i, t}\right)=l_{i}^{G}+2 q_{i}^{G} g_{i, t}$
$0 \leq g_{i, t} \leq g_{i}^{\max }, \forall t$

The profit of producer $i$ at time period $t$ is then given by:

$G P_{i, t}\left(\lambda_{t}, g_{i, t}\right)=\lambda_{t} g_{i, t}-C_{i, t}\left(g_{i, t}\right)$

In line with the majority of relevant works [4], [12]-[15], strategic electricity producers are assumed to exert market power through submitting offers higher than their actual marginal costs (i.e. economic withholding). Following the model employed in these papers, the strategic marginal cost function is expressed by (5), where the value of the decision variable $k_{i, t} \geq 1$ expresses the extent to which producer $i$ misreports its marginal cost at time period $t$.

$S C_{i, t}^{\prime}\left(g_{i, t}\right)=k_{i, t}\left(l_{i}^{G}+2 q_{i}^{G} g_{i, t}\right)$

$k_{i, t}=1$ stands for perfectly competitive generation behavior where producer $i$ reveals its actual marginal cost to the market at $t$, while $k_{i, t}>1$ represents strategic generation behavior where producer $i$ submits higher than its actual marginal cost to the market at $t$. Producer $i$ should decide the value of $k_{i, t}$ by optimally balancing the trade-off between higher market clearing price and lower clearing quantity. More specifically, a higher $k_{i, t}$ will tend to increase the market price at $t$, but at the same time it will tend to reduce the quantity sold by producer $i$, since producers with lower submitted marginal costs may replace $i$ in the merit order and/or the consumers may employ their self-price elasticity or time-shifting flexibility to reduce their demand at $t$.

\section{B. Demand Side}

In line with the model employed in [4]-[6], the benefit or "usefulness" derived by the demand side at each time period is expressed through a quadratic, non-decreasing and concave function (6). The marginal benefit is thus expressed through a linear decreasing function (7) which encapsulates the effect of demand's self-price elasticity. Consumers' willingness to pay decreases as their consumption increases. The maximum price $l_{t}^{D}$ that the consumers are willing to pay represents the value of lost load (VOLL) [7]. The limits in the requested demand level at each time period are expressed by (8). The VOLL, the slope for the marginal benefit function and the maximum demand limit are time-specific parameters, expressing the differentiated preferences of consumers across different time periods [16].

$B_{t}\left(d_{t}\right)=l_{t}^{D} d_{t}-q_{t}^{D}\left(d_{t}\right)^{2}$

$B_{t}^{\prime}\left(d_{t}\right)=l_{t}^{D}-2 q_{t}^{D} d_{t}$

$0 \leq d_{t} \leq d_{t}^{\max }, \forall t$

The time-shifting flexibility of the demand side is expressed by (9)-(11). The variable $d_{t}^{s h}$ represents the change of the demand with respect to the baseline level $d_{t}$ at time period $t$ due to load shifting, taking negative values when demand is moved away from $t$ and positive values when demand is moved towards $t$. Constraint (10) ensures that demand shifting is energy neutral within the examined time horizon i.e. the total size of demand reductions is equal to the total size of demand increases (load recovery), assuming that temporal shifting of demand does not involve energy gains or 
losses. Constraint (11) expresses the limits of demand change at each time period due to load shifting as a ratio $\alpha(0 \leq \alpha \leq$ 1) of the baseline demand; $\alpha=0$ implies that the demand side does not exhibit any time-shifting flexibility, while $\alpha=1$ implies that the whole demand can be shifted in time.

$d_{t}^{\prime}=d_{t}+d_{t}^{s h}, \forall t$

$\sum_{t} d_{t}^{s h}=0$

$-\alpha * d_{t} \leq d_{t}^{s h} \leq \alpha * d_{t}, \forall t$

The utility of the demand side at time period $t$ is given by (12). While the energy payment (second term) depends on the final demand after any potential load shifting, the benefit (first term) is assumed to depend on the baseline demand; this assumption expresses the flexibility of the consumers to shift the operation of some of their loads without compromising the satisfaction they experience.

$D U_{t}\left(\lambda_{t}, d_{t}, d_{t}^{\prime}\right)=B_{t}\left(d_{t}\right)-\lambda_{t} d_{t}^{\prime}$

\section{THEORETICAL ANALYSIS OF IMPACT OF DEMAND SHIFTING ON GENERATION MARKET POWER}

As demonstrated in [8]-[11], the time shifting flexibility of the demand side flattens the demand profile by shaving demand peaks and filling in off-peak demand valleys. Fig. 1 illustrates, in a price-quantity graph, the impact of such demand shifting on the extent of market power exercised by electricity producers, in a simplified market representation involving two periods (peak and off-peak).

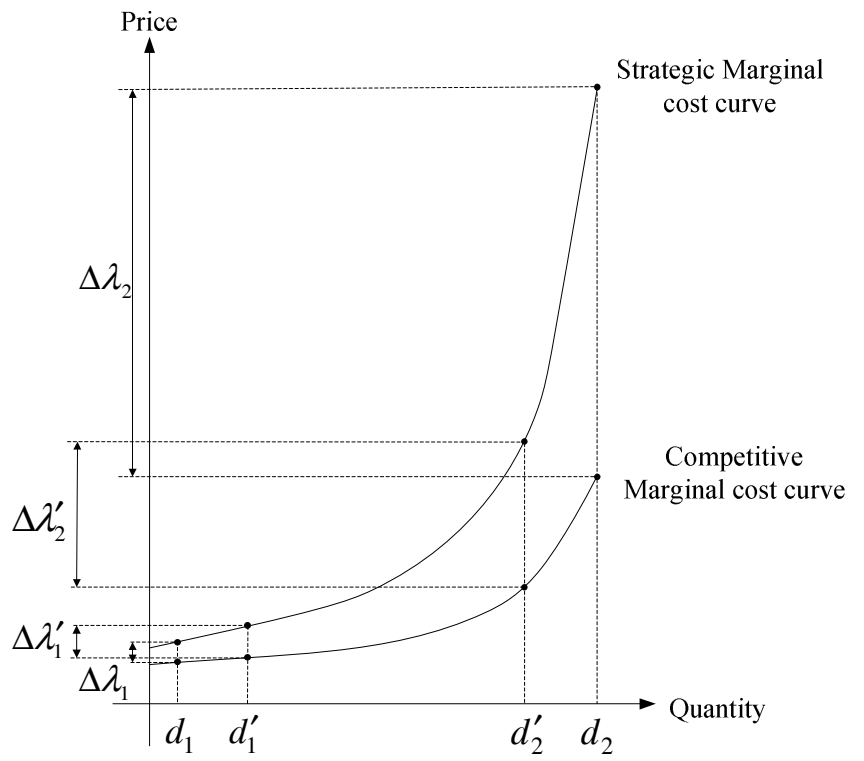

Figure 1. Illusrtation of mitigation effect of demand shifting on the extent of market power exercised by the generation side.

The two curves represent the cumulative marginal cost curves -characterized by increasing slope [1]-[2]- under competitive and strategic generation behavior. The price intercept and slope of the strategic curve are higher than the respective parameters of the competitive curve (Section II-A). Demand shifting shaves the peak demand from $d_{2}$ to $d_{2}^{\prime}$ and increases the off-peak demand from $d_{1}$ to $d_{1}^{\prime}$. Market clearing prices in the respective cases are obtained by the intersections of the marginal cost curves with the vertical demand lines. The price increments $\Delta \lambda$ indicate the increase of the market clearing prices driven by the exercise of market power in the respective cases.

Fig. 1 illustrates that demand shifting reduces the price increment at the peak period from $\Delta \lambda_{2}$ to $\Delta \lambda_{2}^{\prime}$ while it increases it at the off-peak period from $\Delta \lambda_{1}$ to $\Delta \lambda_{1}^{\prime}$. Although the demand shift is energy neutral, i.e. $d_{2}-d_{2}^{\prime}=d_{1}^{\prime}-d_{1}$, the price increment reduction at the peak period is higher than its increase at the off-peak period, i.e. $\Delta \lambda_{2}-\Delta \lambda_{2}^{\prime}>\Delta \lambda_{1}^{\prime}-\Delta \lambda_{1}$, due to the larger slope of the strategic marginal cost curve. This effect also applies to the generation profit increments (as quantitatively explored in Section V) and implies that demand shifting leads to an overall reduction of the extent of market power exerted by the generation side.

\section{MODELING OLIGOPOLISTIC ELECTRICITY MARKETS WITH DEMAND SHIFTING}

\section{A. Decision-Making Problem of Strategic Electricity Producers}

Following the approach widely adopted in relevant work [4], [12]-[15], the decision making process of each strategic electricity producer $i$ is modeled through a bi-level optimization problem. The upper level problem determines the optimal offering strategies $k_{i, t}$ maximizing the producer's profit (13). This problem is subject to the bounds of the offering strategies (14) and the lower level problem (15)-(20). The latter represents the market clearing process at each time period, maximizing the perceived (as strategic producers do not necessarily reveal their actual marginal costs to the market) social welfare (15), and is subject to power balance constraints (16) (the Lagrangian multipliers of which constitute the market clearing prices), generation output limits (17) and the time-coupling operational constraints of the demand side (18)-(20). These two problems are interdependent, since the offering decisions made by the strategic producer at the upper level problem influence the market clearing outcomes in the lower level problem, while the market clearing prices and production quantities obtained in the lower level problem influence the producer's profit in the upper level problem.

(Upper level)

$\max _{\left\{k_{i, t}\right\}} \sum_{t} \lambda_{t} g_{i, t}-C_{i, t}\left(g_{i, t}\right)$

subject to:

$k_{i, t} \geq 1, \forall t$

(Lower level)

$\min _{\left\{g_{i, t}, d_{t}, d_{t}^{s h}\right\}} \sum_{t} k_{i, t} C_{i, t}\left(g_{i, t}\right)+\sum_{i-, t} k_{i-, t} C_{i-, t}\left(g_{i-, t}\right)-$

$\sum_{t} B_{t}\left(d_{t}\right)$

subject to:

$d_{t}+d_{t}^{s h}-\sum_{i} g_{i, t}=0, \forall t$

$0 \leq g_{i, t} \leq g_{i}^{\max }, \forall i, \forall t$ 
$0 \leq d_{t} \leq d_{t}^{\max }, \forall t$

$\sum_{t} d_{t}^{s h}=0$

$-\alpha * d_{t} \leq d_{t}^{s h} \leq \alpha * d_{t}, \forall t$

In order to solve the above bi-level optimization problem, the lower level problem is replaced by its KKT optimality conditions, which is enabled by the continuity and convexity of the lower level problem. This transforms the bi-level problem to a single-level MPEC problem. For space limitation reasons, the derivation of the KKT optimality conditions and the resulting MPEC formulation are not presented here, but they follow the rationale of previous works [12], [14]-[16].

\section{B. Determining Oligolistic Market Equilibrium}

The above formulation expresses the decision making process of a single electricity producer. In order to determine market equilibria resulting from the interaction of multiple independent producers, an iterative diagonalization approach [4], [12]-[13] is employed in this paper. At each iteration, each of the producers solves their MPEC problem -given the offering strategies of the rest of the producers as determined in the previous iteration- until the offering strategies of all producers remain constant with respect to the previous iteration. This state corresponds to a market equilibrium since none of the producers can increase their profits by unilaterally modifying their offering strategies.

\section{CASE STUdies}

The examined case studies involve demonstration of the mitigation effect of demand's time-shifting flexibility on the market power exercised by the generation side in a test market with day-ahead horizon and hourly resolution. This market includes 7 electricity producers and reflects the general generation and demand characteristics of the Great Britain (GB) power system [14]. Different scenarios are examined regarding the time-shifting flexibility of the demand side, as expressed by parameter $\alpha$. For each of these scenarios, two cases have been compared: i) a case of perfectly competitive generation behavior (indicated by the superscript $c$ in the remainder), where all producers behave competitively at all time periods, i.e. $k_{i, t}=1, \forall i, \forall t$, and ii) a case of strategic generation behavior (indicated by the superscript $s$ in the remainder), where the offering strategies of the producers are determined based on the equilibrium model of Section IV.

The developed equilibrium programming model has been coded and solved using the optimization software $\mathrm{FICO}^{\mathrm{TM}}$ Xpress [17] on a computer with a 6-core $3.47 \mathrm{GHz}$ Intel(R) Xeon(R) X5690 processor and 192 GB of RAM. The average computational time required for solving a MPEC problem was around $8 \mathrm{~s}$ and the average number of iterations required for reaching a market equilibrium has been 16 .

In order to quantify the impact of demand shifting on the market power exercised by the generation side, relevant indexes from the literature [4]-[5] are applied. The average Lerner index (AveLI) (21) expresses the average increase of market prices driven by the exercise of market power. Fig. 2 demonstrates that AveLI is reduced with an increasing demand shifting flexibility, implying that the latter limits the ability of strategic generation producers to manipulate market prices (as also qualitatively illustrated in Section III).

Ave $L I=\operatorname{average}_{t} \frac{\lambda_{t}^{s}-\lambda_{t}^{c}}{\lambda_{t}^{s}}(\%)$

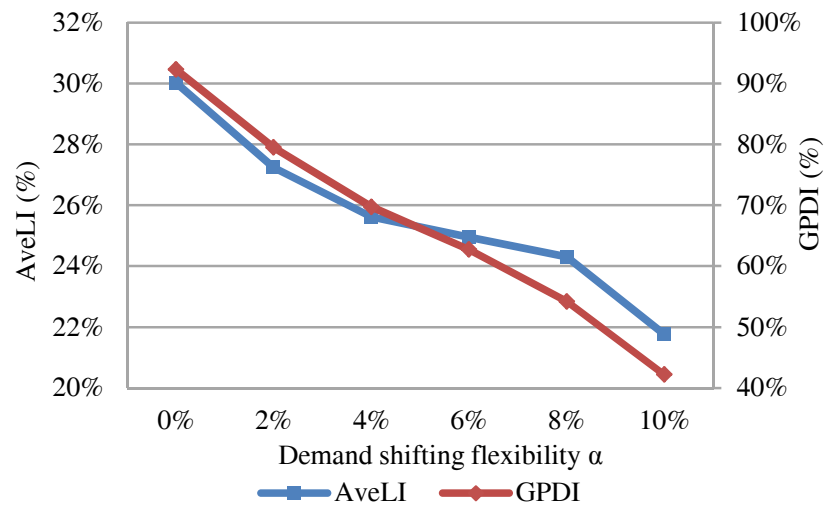

Figure 2. Averge Lerner index and generation profit deviation index for different demand shifting flexibility scenarios

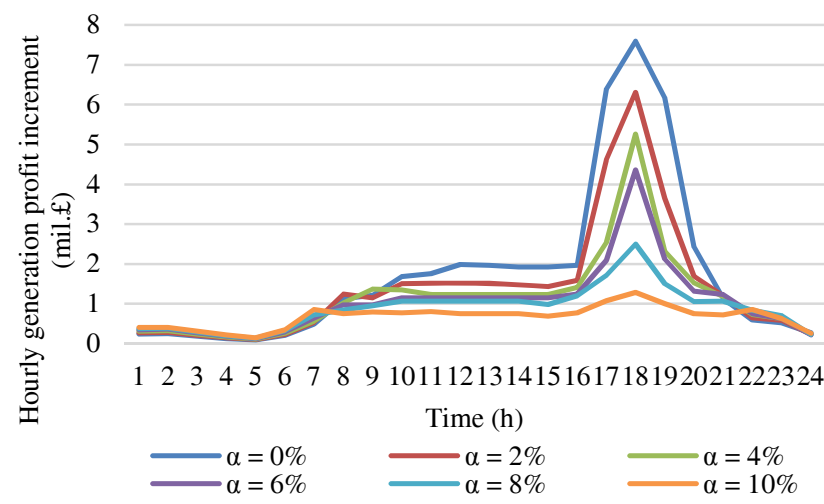

Figure 3. Cumulative hourly increase of generation profit driven by the exercise of market power for different demand shifting flexibility scenarios

This reduction of the generators' market power potential has also an impact on their additional profit driven by the exercise of market power. Fig. 3 presents the cumulative increment of the generation side's hourly profit for different scenarios of demand shifting flexibility. Due to the reason explained in Section III, demand shifting reduces the hourly increase of generation profit during peak hours and increases it during off-peak hours, with the former reduction dominating the latter increase. This effect becomes more evident as the demand shifting flexibility is increased. As a consequence, the total generation profit increment driven by the exercise of market power is substantially reduced. This reduction is also justified by the quantification of the generation profit deviation index (GPDI) (22). Fig. 2 demonstrates that GPDI is reduced with an increasing demand shifting flexibility, implying that the latter reduces the additional generation profit driven by the exercise of market power.

$G P D I=\frac{\sum_{i, t} G P_{i, t}^{S}-\sum_{i, t} G P_{i, t}^{c}}{\sum_{i, t} G P_{i, t}^{C}}(\%)$

Moreover, the reduction of the generation market power has also a beneficial effect on demand side's utility, which is 
justified by the quantification of the demand utility deviation index (DUDI) (23). Fig. 4 demonstrates that (the absolute value of) DUDI is reduced with an increasing demand shifting flexibility, suggesting that the latter reduces the demand utility decrement driven by the exercise of market power, and thus allows consumers to more efficiently secure their economic surplus against electricity producers' gaming behavior.

$D U D I=\frac{\sum_{t} D U_{t}^{S}-\sum_{t} D U_{t}^{c}}{\sum_{t} D U_{t}^{c}}(\%)$

Finally, the reduction of the generation market power potential has also a beneficial impact on social welfare, as justified by the quantification of the market inefficiency index (MII) (24). Fig. 4 demonstrates that (the absolute value of) MII is reduced with an increasing demand shifting flexibility, implying that the latter reduces the social welfare loss driven by the exercise of market power and thus increases the overall efficiency of the market.

$$
M I I=\frac{\sum_{t} S W_{t}^{S}-\sum_{t} S W_{t}^{c}}{\sum_{t} S W_{t}^{c}}(\%)
$$

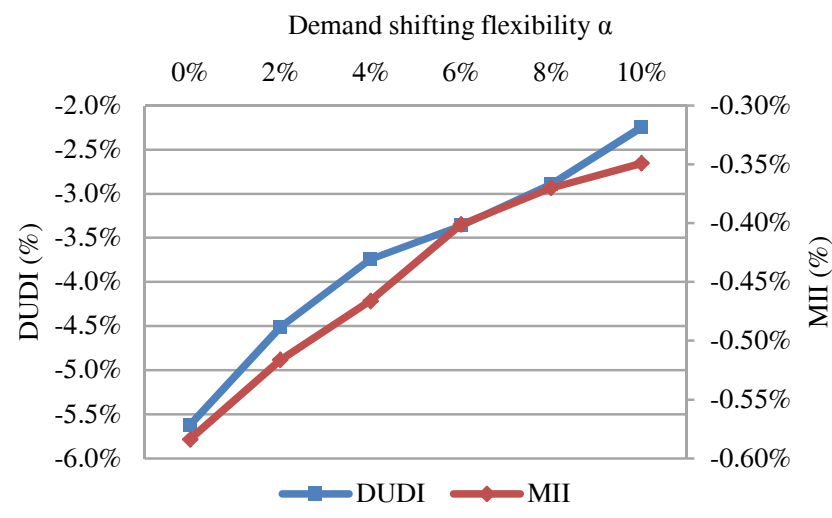

Figure 4. Demand utility deviation index and market inefficiency index for different demand shifting flexibility scenarios

\section{CONCLUSIONS}

This paper has provided both qualitative and quantitative evidence of the value of demand shifting in mitigating market power by strategic electricity producers. Qualitative explanation of this impact has been performed through a simplified two-period example, demonstrating that demand shifting reduces the extent of exerted market power at the peak period and increases it at the off-peak period, with the former reduction being prominently greater than the latter increase and leading to an overall positive impact. Quantitative analysis has been facilitated through an equilibrium programming model of the oligopolistic market setting, taking into account the inter-temporal characteristics of demand shifting.

Case studies with the developed model on a test market with day-ahead horizon and hourly resolution have quantitatively demonstrated the mitigation effects of demand shifting on market power of the generation side, by employing relevant indexes from the literature. An increasing demand shifting flexibility has been shown to i) reduce strategic producers' ability to manipulate market prices, ii) reduce the additional generation profit driven by the exercise of market power, iii) protect consumers' surplus from producers' strategic behavior, and iv) reduce the social welfare loss driven by the exercise of market power.

\section{REFERENCES}

[1] D. Kirschen and G. Strbac, Fundamentals of Power System Economics: John Wiley \& Sons, Ltd, 2004.

[2] D. S. Kirschen, "Demand-side view of electricity markets," IEEE Trans. Power Syst., vol. 18, no. 2, pp. 520-527, May 2003.

[3] T. Ackermann, "Distributed resources and re-regulated electricity markets," Elec. Power Syst. Res., vol. 77, no. 9, pp. 1148-1159, Jul. 2007.

[4] E. Bompard, M. Yuchao, R. Napoli and G. Abrate, "The demand elasticity impacts on the strategic bidding behavior of the electricity producers," IEEE Trans. Power Syst., vol. 22, no. 1, pp. 188-197, Feb. 2007.

[5] E. Bompard, M. Yuchao, R. Napoli, G. Abrate and E. Ragazzi, "The impacts of price responsiveness on strategic equilibrium in competitive electricity markets," Elec. Power Ener. Syst., vol. 29, no. 5, pp. 397407, Jun. 2007.

[6] E. Bompard, R. Napoli and B. Wan, "The effect of the programs for demand response incentives in competitive electricity markets," Eur. Trans. Elec. Power, vol. 19, no. 1, pp. 127-139, Jan. 2009.

[7] P. R. Thimmapuram, J. Kim, A. Botterud and Y. Nam, "Modeling and simulation of price elasticity of demand using an agent-based model," Innov. Smart Grid Tech. (ISGT), Gaithersburg, U.S.A., 2010.

[8] G. Strbac, "Demand side management: Benefits and challenges," Energy Policy, vol. 36, no. 12, pp. 4419-4426, Dec. 2008.

[9] C.-L. Su and D. Kirschen, "Quantifying the effect of demand response in electricity markets," IEEE Trans. Power Syst., vol. 24, no. 3, pp. 1199-1207, Aug. 2009.

[10] D. Papadaskalopoulos and G. Strbac, "Decentralized participation of flexible demand in electricity markets-Part I: Market mechanism," IEEE Trans. Power Syst., vol. 28, no. 4, pp. 3658-3666, Nov. 2013.

[11] Y. Ye, D. Papadaskalopoulos and G. Strbac, "Factoring Flexible Demand Non-Convexities in Electricity Markets," IEEE Trans. Power Syst., vol. 30, no .4, pp. 2090-2099, Jul. 2015.

[12] B. Hobbs, C. Metzler and J. S. Pang, "Strategic gaming analysis for electric power systems: An MPEC approach," IEEE Trans. Power Syst., vol. 15, no. 2, pp. 638-645, May 2000.

[13] J. D. Weber and T. J. Overbye, "An individual welfare maximization algorithm for electricity markets," IEEE Trans. Power Syst., vol. 17, no. 3, pp. 590-596, Aug. 2002.

[14] Y. Ye, D. Papadaskalopoulos and G. Strbac, "An MPEC approach for analyzing the impact of energy storage in imperfect electricity markets," 13th Int. Conf. Eur. Energy Market, Porto, Portugal, 2016.

[15] C. Ruiz and A. J. Conejo, "Pool strategy of a producer with endogenous formation of locational marginal prices," IEEE Trans. Power Syst., vol. 24, no. 4, pp. 1855-1866, Nov. 2009.

[16] S. J. Kazempour, A. J. Conejo and C. Ruiz, "Strategic bidding for a large consumer," IEEE Trans. Power Syst., vol. 30, no. 2, pp. 848-856, Mar. 2015.

[17] FICO XPRESS website, http://www.fico.com/en/Products/DMTools/ Pages/FICO-Xpress-Optimization-Suite.aspx.

\section{BIOGRAPHIES}

Dimitrios Papadaskalopoulos (M'13) is a Research Associate at Imperial College London, London, U.K., since 2013. His current research focuses on the development and application of distributed and market-based approaches for the coordination of operation and planning decisions in power systems, employing optimisation and game theoretic principles.

Yujian Ye (M'12) is currently pursuing the Ph.D. degree at Imperial College London. His current research interests include pricing mechanisms, demand side and energy storage participation, and the application of the game theory in electricity markets.

Goran Strbac (M'95) is a Professor of Electrical Energy Systems at Imperial College London, London, U.K., since 2005. His research interests include electricity system operation, investment and pricing, and integration of renewable generation and distributed energy resources. 